\title{
Calidad de atención en salas de trabajo de parto. Instituciones oficiales. Antioquia-Colombia 1990
}

\author{
María Consuelo Castrillon A.*, Isabel Escobar Saldarriaga**, Socorro Púlido de Lalinde***
}

\begin{abstract}
RESUMEN: Se estudió la calidad de atención que se presta durante el trabajo de parto a mujeres usuarias de los servicios estatales de salud del Departamento de Antioquia, Colombia, durante el primer semestre de 1990.

Se estudiaron tres dimensiones: la atención científico técnica que brinda enfermería, las relaciones interpersonales del equipo de salud con las usuarias y el contexto de los servicios en términos de eficiencia para prestar este servicio.

Las instituciones que mostraron mayor deterioro en recursos, suministros y normatividad técnico administrativa fueron las del tercer nivel de atención, que corresponden a Hospitales Universitarios y de la Seguridad Social. Las acciones científico-técnicas que debe realizar el profesional de enfermería están prácticamente delegadas y con escasa supervisión por falta de este recurso o por que las instituciones han trastocado sus funciones. El porcentaje de gestantes satisfechas con la atención recibida fue mayor en los Hospitales Regionales del SSSA (97\%) y en las UCSS de METROSALUD (92\%).
\end{abstract}

PALABRAS ClAVES: - Calidad de Atención de Salud, Trabajo de Parto, Servicios de Salud, Atención en Salud.

SUMMARY: The quality of attention given in the labor rooms to women affiliated to health institutions was studied in the state of Antioquia, Colombia, during the first semester of 1990.

Three dimensions were studied, the cientific and technical nursing attentions, the interpersonal relations between health groups and clients, and the general view of the units in terms of efficiency to render attention.

The Institutions with greater deterioration in resources, supplies and lacking administrative norms were those of the third level of attention belonging to University and Social Segurity Hospitals. The cientific and technical activities that must be done by the professional nurse are almost completely delegated with minimun supervision; this is due to limited resources or because the institutions have misplaced their functions.

The percentaje of pregnant women satisfied with the attention received was higher in the regional hospitals adscribed to the SSSA (97\%) and to the local units of health in the city $(92 \%)$.

KEY WORDS: - Quality of Health Care, Labor, Health Services, Heath Care.

\section{Introducción}

Se estudió la calidad de atención que se presta durante el trabajo de parto a mujeres usuarias de los servicios institucionales de salud del Departamento de Antioquia, Colombia, durante el primer semestre de 1990.

El diseño teórico parte del concepto de calidad de atención propuesto por Donabedian para quien la calidad es a la vez una propiedad y un juicio que se puede establecer frente a la atención en salud, a partir del estudio de tres dimensiones: la atención científico-técnica, las relaciones interpersonales y el contexto de los servicios.

Aunque la atención en salud se brinda en equipo multidisciplinario es pertinente, según el autor citado, valorar su calidad a través de un sólo tipo profesional. Apoyada en este planteamiento, la investigación evalúa la atención científicotécnica a través de la función asistencial de profesionales de enfermería durante el proceso de: valoración bio-psico-social,

\footnotetext{
* Investigación realizada con financiación del Sistema Universitario de Investigaciones. Universidad de Antioquia.

** Enfermera, MG.SE. Docente Facultad de Enfermería, Universidad de Antioquia.

*** Enfermera, docente Departamento Materno Infantil, Facultad de Enfermería Universidad de Antioquia.
}

diagnóstico de enfermería, planeación de la atención, intervenciones propias de este profesional en esta área y evaluación del plan.

El análisis de la dimensión relaciones interpersonales, buscaba medir el interés que demuestra el personal de salud por las personas a quienes se brinda el servicio y la satisfacción de las usuarias del mismo.

El contexto de los servicios se estudió con base en el concepto condiciones de eficiencia que estudia las características estructurales y de proceso que permiten garantizar la atención al usuario y a la vez evaluar el grado de adecuación de los servicios. Los indicadores estudiados fueron: la planta física, los recursos humanos y materiales, los suministros, las normas y procesimientos, la programación y administración general y el control de salud del personal.

\section{Metodología}

Investigación evaluativa cuali-cuantitativa. Se estudiaron tres tipos de poblaciones, instituciones oficiales, profesionales de enfermería y gestantes que demandaron atención institucional durante el período del estudio (primer semestre de 1990). En esta población se realizó muestreo en forma estratificada por instituciones y proporcional al principio de partos mensuales atendidos. El tamaño muestral fue de 234. 
Las instituciones estudiadas fueron: Servicio Secional de Salud de Antioquia, Seguro Social (ISS León XIII), Instituto Metropolitano de Salud (Metrosalud), Hospital Universitario San Vicente de Paúl y Hospital General de Medellín. En cada institución se realizaron observaciones en las salas de trabajo de parto para estudiar las condiciones de eficiencia mediante escalas ajustadas a partir de las propuestas por la OPS/OMS y las ajustadas para el país. Entrevistas individuales y colectivas a los profesionales de enfermería para estudiar la calidad científico técnica de su función asistencial.

La entrevista colectiva se estructuró a partir del modelo de análisis prospectivo, donde a partir de escenarios diversos que hacen relación a una misma situación, se ayuda al grupo a ubicar el estado de desarrollo de su práctica, el resultado se gráfica para obtener un perfil asistencial y profesional. De la metodología de análisis prospectivo, se desarrollaron sólo dos de las fases de dicho proceso, la fase de exploración y diagnóstico y el análisis en profundidad.

Para cónocer las ópiniones de las gestantes se elaboró una guía de entrevista que fue realizada por estudiantes de antropología. En ella se pidió el relato de su vivencia, la oportunidad en la atención, el trato recibido, la descripción del lugar donde realizó el trabajo de parto y las explicaciones recibidas por parte del personal de salud.

\section{Resultados}

En condiciones de eficiencia las puntuaciones totales con relación al puntaje esperado ( $100 \%$ de condiciones mínimas para prestar un servicio de calidad) por niveles de atención fluctuaron así:

Nivel terciario entre $61.5 \%$ y el $57.2 \%$

Nivel secundario Servicio Seccional de Salud 65.3 a 52.7 y para Metrosalud $61 \%$ a $46.3 \%$

Analizadas en conjunto, las instituciones con mayor deterioro en recursos, suministros y normatividad técnico-administrativa fueron las del tercer nivel de atención, que corresponden a Hospitales Universitarios y de la Seguridad Social donde, además de atender el alto riesgo obstétrico, se realiza docencia e investigación. Dos de estas instituciones registraron una demanda superior a su capacidad instalada durante los tres meses anteriores a las observaciones y una relación cuantitativa entre recurso humano de enfermería y camas hospitalarias inferior a las normas internacionales.

Las deficiencias en suministros, en mantenimiento de equipos y en sistemas de comunicación alteran y entorpecen las funciones científico-técnicas y particularmente las del profesional de enfermería quien debe invertir la mayor parte de su tiempo en llamadas, desplazamientos y elaboración de órdenes para lograr condiciones mínimas de atención. Estas acciones la alejan cada vez más del objeto directo de su práctica que es el usuario del servicio.

Las acciones científico-técnicas del profesional de enfermería están prácticamente delegadas y con escasa supervisión. Esta situación se debe a escasez de personal, demanda superior a la capacidad instalada, deterioro en las condiciones de eficiencia en especial en lo referente a normatividad técnica y administrativa, ausencia de educación en servicios para el personal de enfermería.

El porcentaje de satisfacción de las gestantes fue mayor en los hospitales Regionales del SSSA (97\%) y en las UCSS de Metrosalud (92\%).

Los mayores porcentajes de insatisfacción se presentaron en el nivel terciario con $19 \%$ para el ISS y el $14 \%$ para el Hospital Universitario San vicente de Paúl y el Hospital General de Medellín.

\section{DISCUSION}

La dotación y los recursos institucionales son nacesarios pero no suficientes para prestar una alta calidad de atención en salud. Así el deterioro encontrado en las condiciones de eficiencia de las salas de trabajo de parto de instituciones oficiales del Departamento de Antioquia, es resultado tanto de las carencias presupuestales permanentes (crisis económicas), como del desorden organizativo del sector y de una planeación centrada exclusivamente en indicadores de tipo administrativo (número de egresos, número de acciones).

El desarrollo de conocimientos científicos no marcha al mismo ritmo del desarrollo de la infraestructura de los servicios de salud y el trabajo interdisciplinario no logra articularse en la cotidianeidad de los servicios hospitalarios por los usos del porder y por la formación recibida.

La práctica de separar el padre durante el trabajo de parto y el parto es producto de una normatividad rígida y castrense, legítima el estado de enajenación del padre frente a la crianza de sus hijos que prevalece en nuestro medio y genera situaciones de crísis emocionales en las madres crisis que la afectan a ella y al producto de la gestación. El hallazgo de situaciones sutiles y expresas de violencia reportado por el $12 \%$ de las mujeres entrevistadas, quienes refieren agresiones verbales y comportamentales por parte del personal de salud, exige una mirada que permita replantear las relaciones del personal de salud con los usuarios de los servicios, en términos de reconocimiento y respecto por la dignidad humana y de cara a la construcción de una ética profesional que permita y apoye la humanización de los servicios de salud.

\section{Recomendaciones}

La realización de estudios que midan la calidad de atención utilizando indicadores clínicos y epidemiológicos ayudaría a profundizar en la realidad de atención que reciben las madres que acuden al parto institucional.

Dotar los servicios del personal de enfermería necesario de acuerdo con la demanda y el tipo de riesgos que se atienden, la capacitación en servicio, la elaboración de protocolos de atención para gestantes de alto, mediano y bajo riesgo, la asignación de labores de dotación y mantenimiento a un personal diferente a enfermería y la actualización y puesta en ejecución de normas administrativas y científicastécnicas modernas, permitirá a mediano plazo alcanzar los niveles de calidad mínima esperada para prestar atención según riesgo en los servicios de gineco-obstetricia de nuestras instituciones oficiales. 


\section{BIBLIOGRAFIA}

1. Arias, María Mercedes y Palácio, Francia. Situación de salud materno infantil a nivel nacional, regional y local. Material docente. Universidad de Antioquia. junio de 1987.

2. Colombia, Ministerio de salud. Programa materno infantil, hecho y proyecciones. 3a ed. Bogotá 1986.

3. Colombia, Ministerio de Salud. Plan Nacional de Salud, 1986-1990. Bogotá, 1986.

4. Colombia, Ministerio de Salud. Atención materna y perinatal con criterio de riesgo. Bogotá, 1988.

5. Donabedian, Avedis. La Calidad de la Atención médica: definición y métodos de evaluación. México, La Prensa Médica Mexicana S.A. 1984.
6. Ledy, Susan y Pepper, Mae. Bases conceptuales de la enfermería profesional. OMS/OPS, Filadelfia, J.B: Lippincott Company, 1989.

7. Miller, Mary Ann y Brooten, Dorothy. Enfermería materno-infantil. Períodos del parto, valoración y acciones. España, Interamericana, 1987.

8. Organización Panamericana de la salud (OPS). Condiciones de Eficiencia de los Servicios de Atención Materno-infantil. Washington, 1985.

9. Rico, J. et al Condiciones de eficiencia de los servicios materno-infantiles del Departamento del Valle del Cauca. Cali, 1985.

10. Velásquez, Magdala. Mujer, salud y derechos humanos. EN: La Salud de la Mujer. Medellín, Grupo Salud Colombia, 1989.

11. Villarraga, Liliam y Barroso, Maritza. Maternidad vivida: una alternativa para la salud mental. Bogotá, Universidad Nacional, 1990.

\section{CONGRESOS}

\section{CONGRESO DE LA SOCIEDAD EUROPEA DE MENOPAUSIA}

Madrid - Octubre 29 de 1992

Informes: II Congreso EMS

C-GUZMAN el Bueno, 89

28 OIS Madrid (Spain)

Tel. 34 (1) 5430584 Fax. 34 (1) 5448771

VII CONGRESO INTERNACIONAL DE MENOPAUSIA

Estocolmo Suiza Junio 20 -24 de 1993.

Informes: IMS/93 - C/O Congreso Box 5619

S- 11486 Stockhoim - Sweden

Tel. 46-86126900 - Fax 46-86126292

\section{6 al 11 de Abril de 1992 \\ Informes: Palacio de las Convenciones \\ Apartado Aéreo No. 6046 -La Habana- \\ Cuba Télex 511609 Palco c u \\ Fax: 22-8382/22}

IX CONGRESO DE OBSTETRICIA Y GINECOLOGIA

II CONGRESO DE PERINATOLOGIA Y PLANIFICACION

V REUNION DEL GRUPO REGIONAL DE MEXICO Y CARIBE DE LA FLASOG

\section{CONGRESO DE LA ASOCIACION EUROPEA}

DE GINECOLOGOS Y OBSTETRAS

HELSINKI, Finlandia - Junio 28 - Julio 1 de 1992.

Informes: Sociedad Col. OBST. y GINEC.

\section{CURSO DE ANDROLOGIA}

Barcelona, España - 21 al 23 de Septiembre de 1992

Informes: SOC. COL. OBST y GINEC. 